\title{
MHD Heat and Mass Transfer of an Oscillatory Flow over a Vertical Permeable Plate in a Porous Medium with Chemical Reaction
}

\author{
Mohammad Al Zubi \\ Mechanical Engineering Department, Tafila Technical University, Tafila, Jordan \\ Email: Mohmmad1972@yahoo.com
}

How to cite this paper: Al Zubi, M. (2018) MHD Heat and Mass Transfer of an Oscillatory Flow over a Vertical Permeable Plate in a Porous Medium with Chemical Reaction. Modern Mechanical Engineering, 8, 179-191.

https://doi.org/10.4236/mme.2018.83012

Received: March 1, 2018

Accepted: May 4, 2018

Published: June 13, 2018

Copyright $\odot 2018$ by author and Scientific Research Publishing Inc. This work is licensed under the Creative Commons Attribution International License (CC BY 4.0). http://creativecommons.org/licenses/by/4.0/

\begin{abstract}
The problem of magneto-hydro-dynamic (MHD) mass and heat transfer of an oscillatory fluid in two-dimensional viscous, electrically conducting over an infinite vertical permeable moving plate in a saturated porous medium with the presence of a transverse magnetic field and chemical reaction is analytically presented. The governing equations, momentum, energy, and concentration are solved. Various flow parameters effects on velocity, temperature and concentration fields are discussed. It is found that, the fluid velocity increases with increasing both the permeability and chemical reaction parameters. While, it increases with decreasing the magnetic field parameter. Furthermore, the concentration increases with increasing chemical reaction parameters.
\end{abstract}

\section{Keywords}

Chemical Reaction, Mass and Heat Transfer, Oscillatory Flow, Porous Medium

\section{Introduction}

The study of the area of (MHD) mass and heat transfer problems combined with chemical reaction in the presence of magnetic field through porous medium has importance in many practical applications in the areas of engineering and science. This phenomenon plays a very important role in many science and technology branches such as chemical industry, geothermal system, nuclear reactors cooling, petroleum industry, and packed-bed catalytic reactors. Due to this wide range of applications, many authors presented solutions for this type of flow with the presence of a magnetic field. Ibrahim et al. (2008) [1], reported an 
analytical solution for the problem of mass and heat transfer of an electrically conducting, heat generation/absorption, Newtonian, laminar, and viscous flow fluid on a continuously vertical permeable surface with the presence of a radiation, Vajravelu (1986) [2], studied the exact solutions of heat transfer and hydro-magnetic boundary-layer flow over a moving continuous flat surface with internal heat generation/absorption and uniform suction, this type of flow represents a new class of boundary-layer problems, with substantially solutions different from those for boundary layer flow on a finite length flat surface. Rout et al. (2013) [3], investigated the combined effects of internal heat generation and a convective boundary condition and chemical reaction on the laminar boundary layer MHD mass and heat transfer flow over a moving vertical flat plate. A stream of cold fluid flows over the upper surface with heat source and chemical reaction and the lower surface of the plate is in contact with a hot fluid. Modather et al. (2009) [4], presented numerical solutions for the governing energy, momentum, angular momentum, and concentration equations of a mass and heat transfer problem of an oscillatory two dimensional micro polar, viscous, electrically conducting fluid over an infinite permeable moving plate in a saturated porous medium with the presence of a transverse magnetic field. Reddy et al. (2013) [5], discussed the mass and heat transfer on magneto hydrodynamic unsteady visco elastic second order Rivlin-Erickson fluid past an impulsively started vertical plate with the presence of a foreign mass and constant mass flux taking into account the viscous dissipative heat at the plate under the influence of a uniform transverse magnetic field in the presence of chemical reaction. Das et al. (1994) [6], studied the exact solution to the flow due to impulsive motion of an infinite vertical plate in its own plane in the presence of species concentration, chemical reaction of first order and constant heat flux at the plate by the Laplace transform technique. Ahmed and Das (2013) [7], presented the effects of chemical reaction and thermal radiation on unsteady MHD mass transfer flow past a semi-infinite vertical porous plate, which is embedded in porous medium in a slip flow regime with variable suction. Muthucumaraswamy (2001) [8], presented a finite-difference solution of the transient natural convection flow of a viscous incompressible fluid past an impulsively started semi-infinite plate with uniform heat and mass flux, in the presence of homogeneous chemical reaction of the first order . Muthucumaraswamy (2002) [9], presented mass and heat transfer effects on a continuously moving isothermal vertical surface with uniform suction. Kandasamy et al. (2006) [10], studied the chemical reaction, mass and heat transfer effect on the boundary layer flow over a porous wedge with heat radiation with the presence of suction or injection. Anjali Devi and Kandasamy (2002) [11], presented an approximate solution for the steady laminar boundary-layer viscous and incompressible flow over a wall of the wedge with suction or injection with the presence of species concentration and mass diffusion solving using Runge-Kutta Gill's method. Chamkha (2003) [12], presented an analytical solution for mass and heat transfer by laminar flow 
of a viscous, Newtonian, electrically conducting and heat generating/absorbing fluid on a continuously moving vertical permeable surface with the presence of first-order chemical reaction and magnetic field. Kandasamy et al. (2005) [13], carried out an analysis to obtain the nonlinear MHD flow with mass and heat transfer characteristics of a viscous, incompressible, electrically conducting and Boussinesq fluid on a vertical stretching surface with thermal stratification and chemical reaction effect. Khadrawi and Al-Odat (2005) [14], studied analytically the transient MHD free convection flows over a semi-infinite moving vertical permeable flat plate embedded a porous medium with uniform suction using the Laplace transformation technique. Chamkha, (1997) [15], studied the laminar, steady, free convection problem of flow over a vertical porous surface with the presence of heat generation or absorption and magnetic field. Aldoss et al., (1995) [16], have studied magnetohydrodynamic mixed convection flow about a vertical plate embedded in porous medium with the presence of a magnetic field.

Therefore, in this study the case of MHD mass and heat transfer of an Oscillatory flow over a permeable vertical-plate with a porous medium and chemical reaction, will be investigated. In addition, the effects of several flow parameters on the thermal and hydrodynamics behavior will be analyzed.

\section{Mathematical Formulation}

Consider a two-dimensional, unsteady, laminar non-Darcian mixed convection flow of an incompressible, viscous, electrically conducting fluid over a permeable infinite vertical plate with a saturated porous medium. A magnetic field of strength $\left(B_{0}\right)$ is applied vertically to the surface, neglecting the effect of the induced magnetic field. $x^{\prime}$ axis is taken upward direction along the planar surface while $y^{\prime}$ axis is taken normal to the surface. Due to the assumption that the plane surface is infinite, the flow variables are functions of $y^{\prime}$ and time $t^{\prime}$ only. The fluid and the plate are assumed to be at rest initially, then, as time $t>0$, the whole system is allowed to move with a constant velocity. The plate temperature is raised suddenly to $T_{w}$ at $t=0$ and maintained constant after that.

[17] [18] [19] give the governing equations for such a motion:

$$
\begin{gathered}
\frac{\partial v^{\prime}}{\partial y^{\prime}}=0 \\
\frac{\partial u^{\prime}}{\partial t^{\prime}}+v^{\prime} \frac{\partial u^{\prime}}{\partial y^{\prime}}=v \frac{\partial^{2} u^{\prime}}{\partial y^{\prime 2}}+v_{r} \frac{\partial^{2} u^{\prime}}{\partial y^{\prime 2}}+g \beta_{T}\left(T-T_{\infty}\right)+g \beta_{C}\left(C-C_{\infty}\right) \\
-\frac{\sigma B_{0}^{2}}{\rho} u^{\prime}-\frac{v}{K^{\prime}} u^{\prime}-\frac{v_{r}}{K^{\prime}} u^{\prime} \\
\frac{\partial T}{\partial t^{\prime}}+v^{\prime} \frac{\partial T}{\partial y^{\prime}}=\alpha \frac{\partial^{2} T}{\partial y^{\prime 2}} \\
\frac{\partial C}{\partial t^{\prime}}+v^{\prime} \frac{\partial C}{\partial y^{\prime}}=D \frac{\partial^{2} C}{\partial y^{\prime 2}}+\gamma_{1}^{\prime}\left(C-C_{\infty}\right)
\end{gathered}
$$


where $\left(u^{\prime}, v^{\prime}\right)$ are the components of velocity at any point $\left(x^{\prime}, y^{\prime}\right) ; T$ is the temperature of the fluid; and $C$ is the mass concentration of the species in the flow. $\rho, v_{r}, v, g, \beta_{C}, \beta_{T}, \sigma, K^{\prime}, \gamma, \alpha, D$ and $\gamma_{1}^{\prime}$ are the density, kinematic rotational viscosity, kinematic viscosity, gravitational acceleration, volumetric mass expansion and volumetric thermal expansion coefficients of the fluid, electrical conductivity of the fluid, medium permeability, micro inertia per unit mass, spin gradient viscosity, thermal diffusivity, molecular diffusivity, and the dimensional chemical reaction parameter, respectively.

The appropriate boundary conditions for the problem are:

$$
\begin{aligned}
& u^{\prime}=u_{p}^{\prime}, T=T_{\infty}+\varepsilon\left(T_{w}-T_{\infty}\right) \mathrm{e}^{n^{t^{\prime}}}, \\
& C=C_{\infty}+\varepsilon\left(C_{w}-C_{\infty}\right) \mathrm{e}^{n^{\prime t^{\prime}}} \text { at } y^{\prime}=0 \\
& u^{\prime} \rightarrow 0, T \rightarrow T_{\infty}, C \rightarrow C_{\infty} \text { as } y^{\prime} \rightarrow \infty
\end{aligned}
$$

Integrating the continuity Equation (1), we get

$$
v^{\prime}=-V_{0}
$$

where $V_{0}$ is a suction velocity scale, which has a non-zero positive constant, $T_{\infty}$ and $C_{\infty}$ are temperature and mass concentration at the free stream

It is convenient to employ the following dimensionless variables

$$
\begin{aligned}
& u=\frac{u^{\prime}}{U_{0}}, \quad t=\frac{V_{0}^{2}}{v} t^{\prime}, \quad \theta=\frac{T-T_{\infty}}{T_{w}-T_{\infty}}, \quad \phi=\frac{C-C_{\infty}}{C_{w}-C_{\infty}}, \\
& v=\frac{v^{\prime}}{V_{0}}, \quad G_{r}=\frac{v g \beta_{T}\left(T_{w}-T_{\infty}\right)}{U_{0} V_{0}^{2}}, \quad G_{m}=\frac{v g \beta_{C}\left(C_{w}-C_{\infty}\right)}{U_{0} V_{0}^{2}}, \\
& y=\frac{V_{0}}{v} y^{\prime}, \quad M=\frac{\sigma \beta_{0}^{2} v}{\rho V_{0}^{2}}, \quad \beta=\frac{v_{r}}{v}, \quad \lambda=\frac{K^{\prime} V_{0}^{2}}{v^{2}}, \quad n^{\prime}=\frac{V_{0}^{2}}{v} n, \\
& P_{r}=\frac{v}{\alpha}, \quad S_{c}=\frac{v}{D}, \quad \gamma_{1}=\frac{v \gamma_{1}^{\prime}}{V_{0}^{2}}, \quad u_{p}^{\prime}=U_{0} U_{p}
\end{aligned}
$$

where $U_{0}$ is a free stream velocity scale, $U_{P}$ is the dimensionless plate velocity and $\beta$ denotes the dimensionless viscosity. $P_{r}, S_{c}, M, G_{r}, G_{m}, \lambda$ and $\gamma_{1}$ are the Prandtl number, Schmidt number, magnetic field parameter, Grashof number for heat transfer, Grashof number for mass transfer, permeability parameter, and the dimensionless chemical reaction parameter, respectively.

With the help of Equation (5), Equations (1) - (6) reduce to the following initial-value problems:

$$
\begin{gathered}
\frac{\partial u}{\partial t}-\frac{\partial u}{\partial y}=(1+\beta) \frac{\partial^{2} u}{\partial y^{2}}+G_{r} \theta+G_{m} \phi-M u-\frac{1+\beta}{\lambda} u \\
\frac{\partial \theta}{\partial t}-\frac{\partial \theta}{\partial y}=\frac{1}{p_{r}} \frac{\partial^{2} \theta}{\partial y^{2}} \\
\frac{\partial \phi}{\partial t}-\frac{\partial \phi}{\partial y}=\frac{1}{S_{c}} \frac{\partial^{2} \phi}{\partial y^{2}}+\gamma_{1} \phi
\end{gathered}
$$

With the following dimensionless boundary conditions:

$$
\begin{aligned}
& u=U_{p}, \quad \theta=\varepsilon \mathrm{e}^{n t}, \quad \phi=\varepsilon \mathrm{e}^{n t} \text { at } y=0 \\
& u \rightarrow 0, \quad \theta \rightarrow 0, \quad \phi \rightarrow 0 \text { as } y \rightarrow \infty
\end{aligned}
$$


Maple and Mathematica software are used to solve and draw the results of the simulation in this paper.

Equations (8) - (10) subjected to the boundary conditions (11), we may use the following linear transformations for low values of $\varepsilon$

$$
\begin{aligned}
& u(y, t)=u_{0}(y)+\varepsilon \mathrm{e}^{n t} u_{1}(y)+O\left(\varepsilon^{2}\right) \\
& \theta(y, t)=\theta_{0}(y)+\varepsilon \mathrm{e}^{n t} \theta_{1}(y)+O\left(\varepsilon^{2}\right) \\
& \phi(y, t)=\phi_{0}(y)+\varepsilon \mathrm{e}^{n t} \phi_{1}(y)+O\left(\varepsilon^{2}\right)
\end{aligned}
$$

Substituting the expressions (12) into Equations (8) - (10), and equating the harmonic and non harmonic terms and neglecting the coefficient of $O\left(\varepsilon^{2}\right)$ we have:

$$
\begin{gathered}
(1+\beta) u_{0}^{\prime \prime}+u_{0}^{\prime}-\frac{1+\beta}{\lambda} u_{0}-M u_{0}=-G_{r} \theta_{0}-G_{m} \phi_{0} \\
(1+\beta) u_{1}^{\prime \prime}+u_{1}^{\prime}+n u_{1}-\frac{1+\beta}{\lambda} u_{1}-M u_{1}=-G_{r} \theta_{1}-G_{m} \phi_{1} \\
\theta_{0}^{\prime \prime}+p_{r} \theta_{0}^{\prime}=0.0 \\
\theta_{1}^{\prime \prime}+p_{r} \theta_{1}^{\prime}+n p_{r} \theta_{1}=0.0 \\
\phi_{0}^{\prime \prime}+S_{c} \phi_{0}^{\prime}+S_{c} \gamma_{1} \phi_{0}=0.0 \\
\phi_{1}^{\prime \prime}+S_{c} \phi_{1}^{\prime}+S_{c}\left(n+\gamma_{1}\right) \phi_{1}=0.0
\end{gathered}
$$

With the following boundary conditions:

$$
\begin{array}{ll}
u_{0}=U_{p}, & u_{1}=0.0, \quad \theta_{0}=1, \quad \theta_{1}=1, \quad \phi_{0}=1, \quad \phi_{1}=1 \text { at } y=0 \\
u_{0}=0.0, & u_{1}=0.0, \quad \theta_{0}=0.0, \quad \theta_{1}=0.0, \quad \phi_{0}=0.0, \quad \phi_{1}=0.0 \text { at } y \rightarrow \infty
\end{array}
$$

The solution of Equations (13) to (18) under the boundary condition (19) are given by:

$$
\begin{gathered}
\theta_{0}(y)=c_{5}+c_{6} \mathrm{e}^{-P_{r} y} \\
\theta_{1}(y)=c_{11} \mathrm{e}^{s_{1} y}+c_{12} \mathrm{e}^{s_{2} y} \\
\phi_{0}(y)=c_{3} \mathrm{e}^{h_{1} y}+c_{4} \mathrm{e}^{h_{2} y} \\
\phi_{1}(y)=c_{9} \mathrm{e}^{s_{3} y}+c_{10} \mathrm{e}^{s_{4} y} \\
u_{0}(y)=c_{2} \mathrm{e}^{-h_{3} y}+c_{1} \mathrm{e}^{-h_{4} y} \\
+\frac{1}{a_{4}}\left(\left(-\left(\frac{c_{3} G_{m} \mathrm{e}^{h_{5} y}}{h_{5}}+\frac{c_{4} G_{m} \mathrm{e}^{h_{6} y}}{h_{6}}+\frac{c_{5} G_{r} \mathrm{e}^{h_{3} y}}{h_{3}}+\frac{c_{6} G_{r} \mathrm{e}^{h_{7} y}}{h_{7}}\right) \mathrm{e}^{\frac{a_{4} y}{a_{1}}}\right.\right. \\
\left.\left.+\frac{c_{3} G_{m} e^{h_{8} y}}{h_{8}}+\frac{c_{4} G_{m} e^{h_{9} y}}{h_{9}}+\frac{c_{5} G_{r} e^{h_{4} y}}{h_{4}}+\frac{c_{6} G_{r} e^{h_{10} y}}{h_{10}}\right) \mathrm{e}^{-h_{4} y}\right) \\
\left.u_{1}(y)=c_{8} \mathrm{e}^{b_{1} y}+c_{7} \mathrm{e}^{b_{2} y}\right) \\
-\frac{1}{a_{5}}\left(\left(\frac{c_{9} G_{m} \mathrm{e}^{b_{3} y}}{b_{3}}+\frac{c_{10} G_{m} \mathrm{e}^{b_{4} y}}{b_{4}}+\frac{c_{11} G_{r} \mathrm{e}^{b_{5} y}}{b_{5}}+\frac{c_{12} G_{r} \mathrm{e}^{b_{6} y}}{b_{6}}\right) \mathrm{e}^{\frac{a_{5} y}{a_{1} y}}\right. \\
\left.\left.-\frac{c_{9} G_{m} \mathrm{e}^{b_{7} y}}{b_{7}}-\frac{c_{10} G_{m} \mathrm{e}^{b_{8} y}}{b_{8}}-\frac{c_{11} G_{r} \mathrm{e}^{b 9 y}}{b_{9}}-\frac{c_{12} G_{r} \mathrm{e}^{b_{10} y}}{b_{10}}\right) \mathrm{e}^{b_{2} y}\right)
\end{gathered}
$$


where:

$$
\begin{gathered}
h_{1}=-\frac{1}{2} S_{c}+\frac{1}{2} \sqrt{S_{c}^{2}-4 a_{3}}, \quad h_{2}=-\frac{1}{2} S_{c}-\frac{1}{2} \sqrt{S_{c}^{2}-4 a_{3}}, \quad h_{3}=\frac{1}{2 a_{1}}-\frac{1}{2} \frac{a_{4}}{a_{1}}, \\
h_{4}=\frac{1}{2 a_{1}}+\frac{1}{2} \frac{a_{4}}{a_{1}}, \quad h_{5}=h_{1}+h_{3}, \quad h_{6}=h_{2}+h_{3}, \quad h_{7}=h_{3}-p_{r}, \quad h_{8}=h_{1}+h_{4}, \\
h_{9}=h_{2}+h_{4}, \quad h_{10}=h_{4}-p_{r}, \quad s_{1}=-\frac{1}{2} p_{r}+\frac{1}{2} \sqrt{p_{r}^{2}-4 m_{2}}, \\
s_{2}=-\frac{1}{2} p_{r}-\frac{1}{2} \sqrt{p_{r}^{2}-4 m_{2}}, \quad s_{3}=-\frac{1}{2} s_{c}+\frac{1}{2} \sqrt{s_{c}^{2}-4 m_{3}}, \\
s_{4}=-\frac{1}{2} s_{c}-\frac{1}{2} \sqrt{s_{c}^{2}-4 m_{3}}, a_{1}=1+\beta, \quad a_{2}=M+\frac{a_{1}}{\lambda}, \quad a_{3}=S_{c} \gamma_{1}, \\
a_{4}=\sqrt{1+4 a_{1} a_{2}}, \quad a_{5}=\sqrt{1-4 a_{1} m_{1}}, \quad b_{1}=\frac{-1}{2 a_{1}}+\frac{1}{2} \frac{a_{5}}{a_{1}}, \quad b_{2}=\frac{-1}{2 a_{1}}-\frac{1}{2} \frac{a_{5}}{a_{1}}, \\
b_{3}=s_{3}-b_{1}, \quad b_{4}=s_{4}-b_{1}, \quad b_{5}=s_{1}-b_{1}, \quad b_{6}=s_{2}-b_{1}, \quad b_{7}=s_{3}-b_{2}, \quad b_{8}=s_{4}-b_{2}, \\
b_{9}=s_{1}-b_{2}, \quad b_{10}=s_{2}-b_{2}, \quad m_{1}=n-a_{2}, \quad m_{2}=n p_{r}, \quad m_{3}=S_{c}\left(n+\gamma_{1}\right)
\end{gathered}
$$

where $c_{i}$ constants are the constants of integration, they are not included here for the sake of brevity.

\section{Results and Discussion}

An analytical solution for the problem of magneto-hydro-dynamic mass and heat Transfer of an Oscillatory Flow over a Vertical Permeable Plate in a Porous Medium with Chemical reactions was investigated. The analytical solutions are evaluated numerically for various parameters involved in the problem. Numerical calculations for non-dimensional temperature, velocity and concentration with different values of physical parameters such as Magnetic parameter, thermal Grashof number, Permeability parameter, Solutal Grashof number, Prandtl number, Schmidt number and chemical reaction parameter.

Figure 1 shows the effect of permeability parameter $\lambda$ on the velocity. It was observed that the fluid velocity increases as the permeability parameter increases. This is because of the presence of the porous medium in the flow presents resistance to flow. So, the resulting resistive force tends to slow the motion of the fluid along the plate surface.

Figure 2 and Figure 3 illustrate the behavior of velocity and concentration for different values of chemical reaction parameter $\gamma_{1}$. It can be noticed that the velocity and concentration increase with increasing the chemical reaction parameter because the chemical reaction enhances momentum transfer and therefore accelerates the flow.

Figure 4 shows the concentration distribution for different values of Schmidt number $S_{c}$ The concentration decreases as the Schmidt number increases. Causing the concentration buoyancy effects to decrease, yielding a reduction in the fluid velocity. The reduction in the velocity and concentration profiles is 


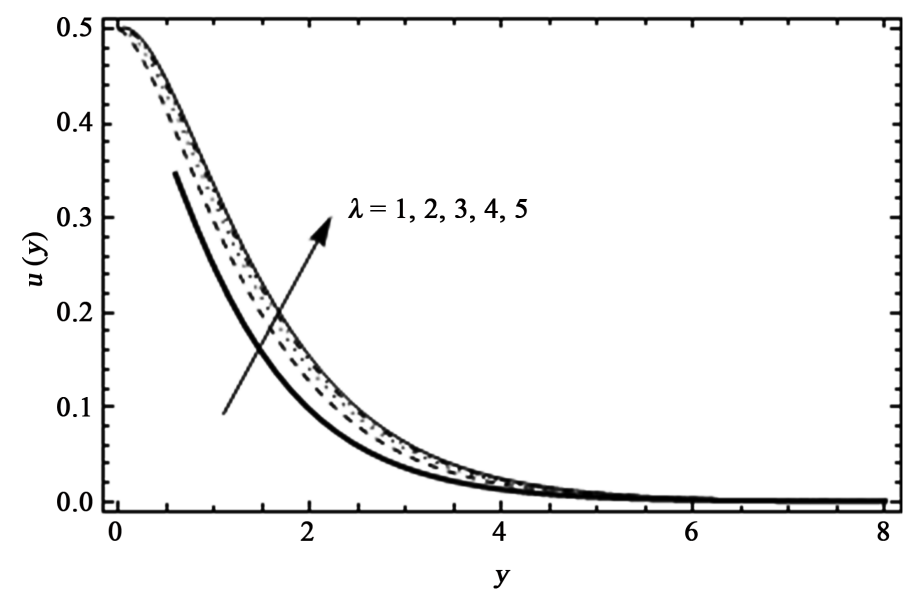

Figure 1. Velocity distribution for different values of $\lambda$ for $G_{r}=2.0, G_{m}=1.0, \beta=1.0$, $M=2.0, \eta=1.0, P_{r}=1.0, S_{c}=2.0, \gamma_{1}=0.2, n=0.1, U=0.5, n_{1}=0.5, \varepsilon=0.01, t=1.0$.

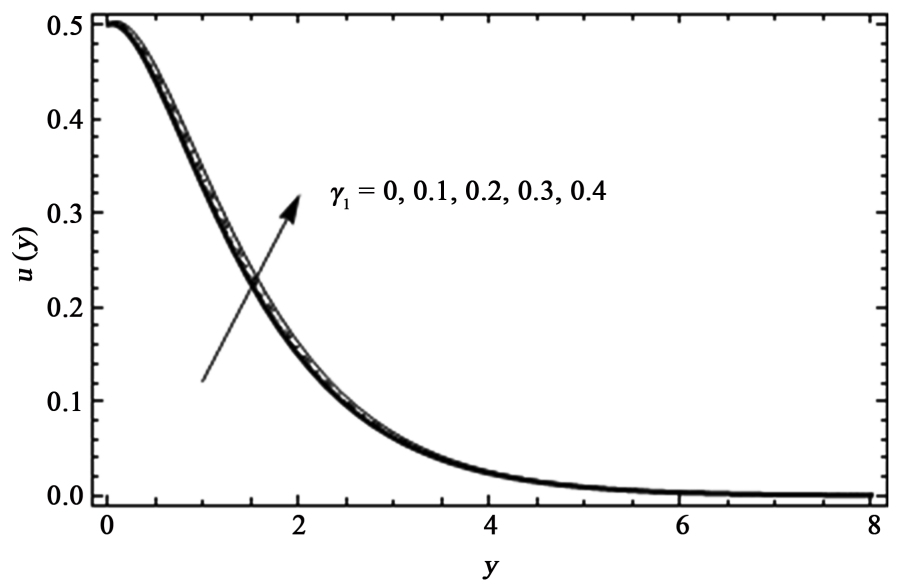

Figure 2. Velocity distribution for different values of $\gamma_{1}$ for $\lambda=5.0, G_{r}=2.0, G_{m}=1.0$, $\beta=1.0, M=2.0, \eta=1.0, P_{r}=1.0, S_{c}=2.0, n=0.1, U=0.5, n_{1}=0.5, \varepsilon=0.01, t=1.0$.

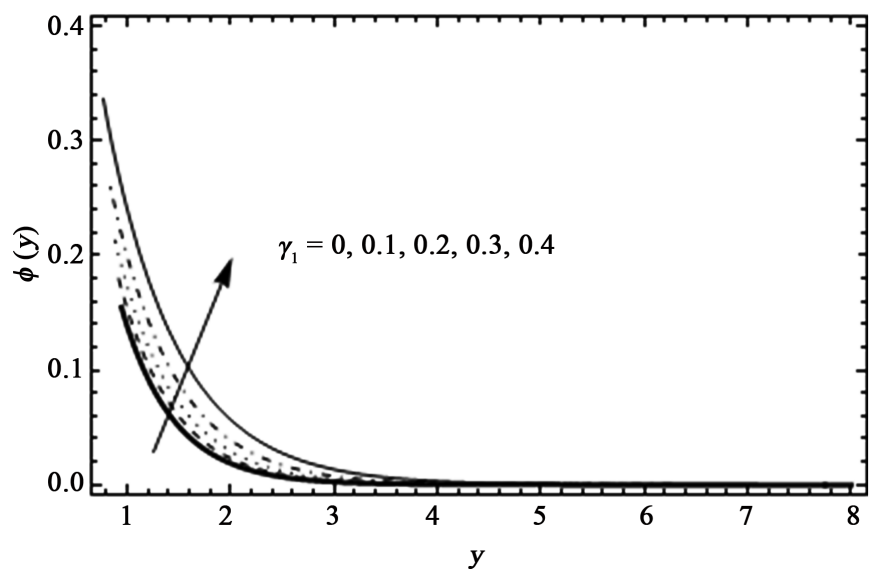

Figure 3. Concentration distribution for different values of $\gamma_{1}$ for $\lambda=5.0, G_{r}=2.0$, $G_{m}=1.0, \quad \beta=1.0, \quad M=2.0, \quad \eta=1.0, P_{r}=1.0, S_{c}=2.0, \quad n=0.1, \quad U=0.5$, $n_{1}=0.5, \varepsilon=0.01, t=1.0$. 


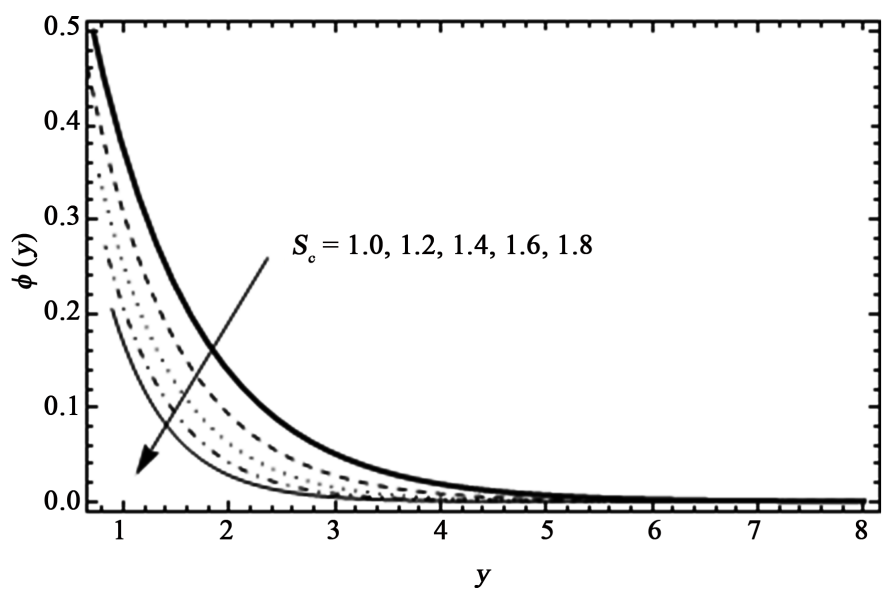

Figure 4. Concentration distribution for different values of $S_{\mathrm{c}}$ for $\lambda=5.0, G_{r}=2.0$,

$G_{m}=1.0, \quad \beta=1.0, \quad M=2.0, \quad \eta=1.0, \quad \eta=1.0, \quad \eta=1.0, \quad \eta=1.0, \quad U=0.5$, $U=0.5, \varepsilon=0.01, t=1.0$.

accompanied by simultaneous reduction in the velocity and concentration boundary layers.

Figure 5 illustrates the velocity distribution for different values of magnetic field parameter $M$. it can be seen that the velocity distribution increases with the decreases of magnetic field parameter. This is because the application of a magnetic field gives rise to a flow resistive force.

Figure 6 and Figure 7 show the unsteady velocity and concentration distribution. It can be seen that the fluid velocity and concentration increase with time. Near the surface, the velocity profile increases to the maximum and then decreases until it reaches an asymptotic value. Figure 8 shows unsteady temperature distribution. It can be noticed that the temperature increases as time increases. Moreover, as time decreases, the thermal boundary layer thickness decreases and the temperature gradient at the wall increases and hence the heat transfer rate increases. The temperature profile is large at the surface and then decreases far away from the wall until it reaches an asymptotic value.

Figure 9 and Figure 10 show a plot of the velocity and temperature profiles versus different values of Prandtl number $P_{r}$. It can be observed that the velocity and temperature of the flow field decrease as Prandtl number $P_{r}$ increases. Thus higher Prandtl number $P_{r}$ leads to faster cooling of the plate.

Figure 11 shows the velocity profile for different values of thermal Grashof number $G_{r}$, it can be found that an increse in the thermal Grashof number $G_{r}$ leads to a rise in the velocity because of the enhancement in buoyancy force.

Figure 12 shows the velocity profile for different values of solutal Grashof number $G_{m}$. The velocity distribution attains a maximum value in the region and then decreases to approach a free stream value. This is evident in the incraese in the value of velocity as solutal Grashof number $G_{m}$ increases.

Figure 13 shows the effect of Schmidt number $S_{c}$ on the velocity. the fluid velocity decreases as the Schmidt number increases, the reduction in velocity 


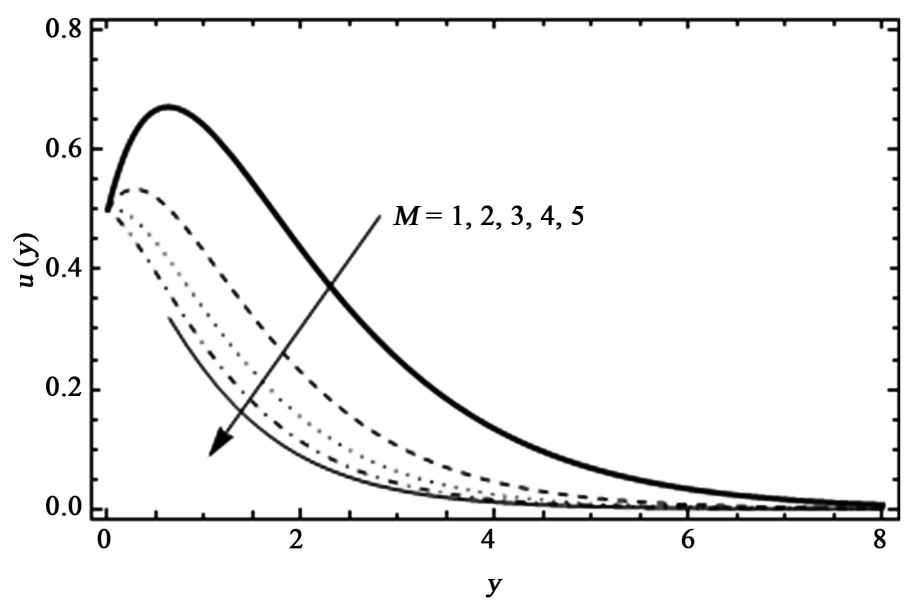

Figure 5. Velocity distribution for different values of $M$ for $\lambda=5.0, G_{r}=2.0, G_{m}=1.0$, $\beta=1.0, \eta=1.0, P_{r}=1.0, S_{c}=2.0, \gamma_{1}=0.2, n=0.1, U=0.5, n_{1}=0.5, \varepsilon=0.01, t=1.0$.

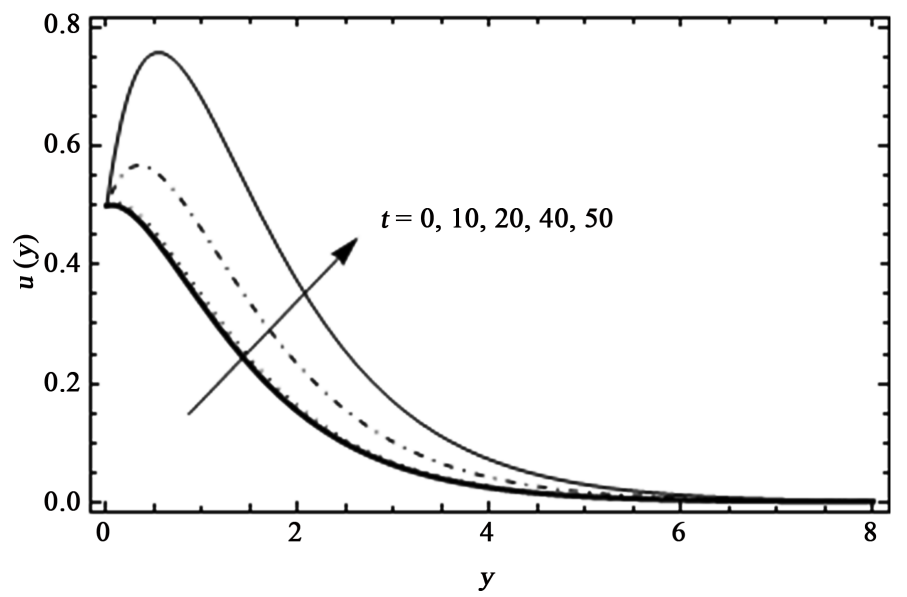

Figure 6. Unsteady velocity distribution for $\lambda=5.0, G_{r}=2.0, G_{m}=1.0, \beta=1.0, M=2.0$, $\eta=1.0, P_{r}=1.0, S_{c}=2.0, \gamma_{1}=0.2, n=0.1, U=0.5, n_{1}=0.5, \varepsilon=0.01$.

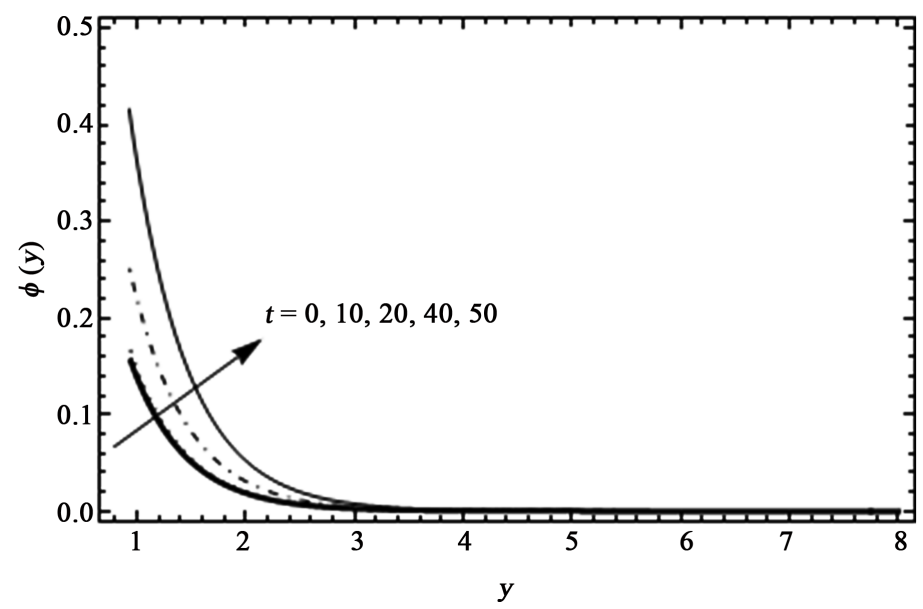

Figure 7. Unsteady concentration distribution for $\lambda=5.0, G_{r}=2.0, G_{m}=1.0, \beta=1.0$, $M=2.0, \eta=1.0, P_{r}=1.0, S_{c}=2.0, \gamma_{1}=0.2, n=0.1, U=0.5, n_{1}=0.5, \varepsilon=0.01$. 


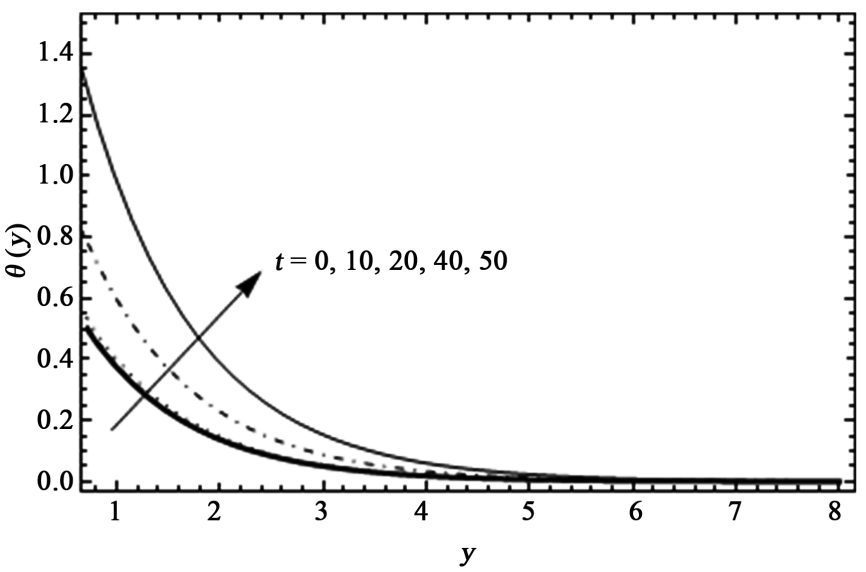

Figure 8. Unsteady temperature distribution for $\lambda=5.0, G_{r}=2.0, G_{m}=1.0, \beta=1.0$, $M=2.0, \eta=1.0, P_{r}=1.0, S_{c}=2.0, \gamma_{1}=0.2, n=0.1, U=0.5, n_{1}=0.5, \varepsilon=0.01$.

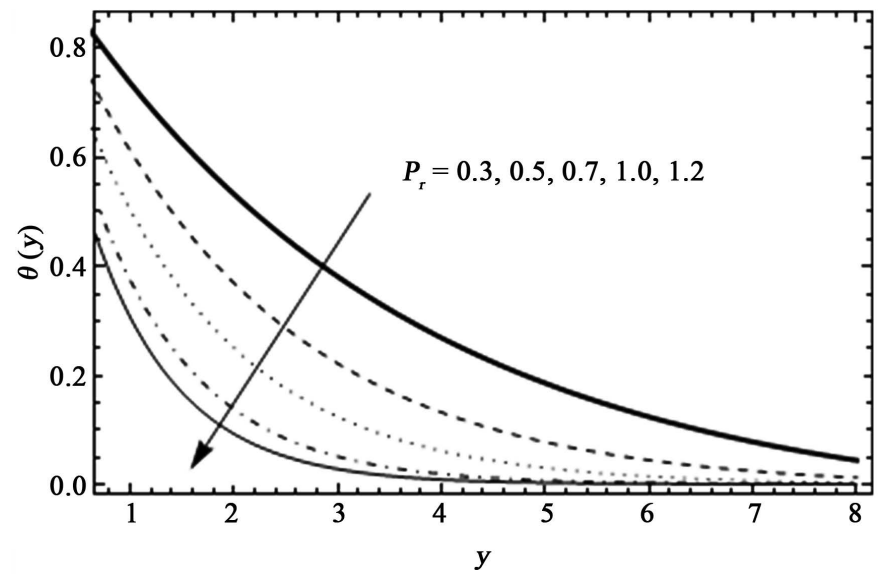

Figure 9. Temperature profile for different values of $P_{r}$ $\lambda=5.0, G_{r}=2.0, G_{m}=1.0, \beta=1.0, M=2.0, \eta=1.0, S_{c}=2.0$, $\gamma_{1}=0.2, n=0.1, U=0.5, n_{1}=0.5, \varepsilon=0.01, t=1.0$.

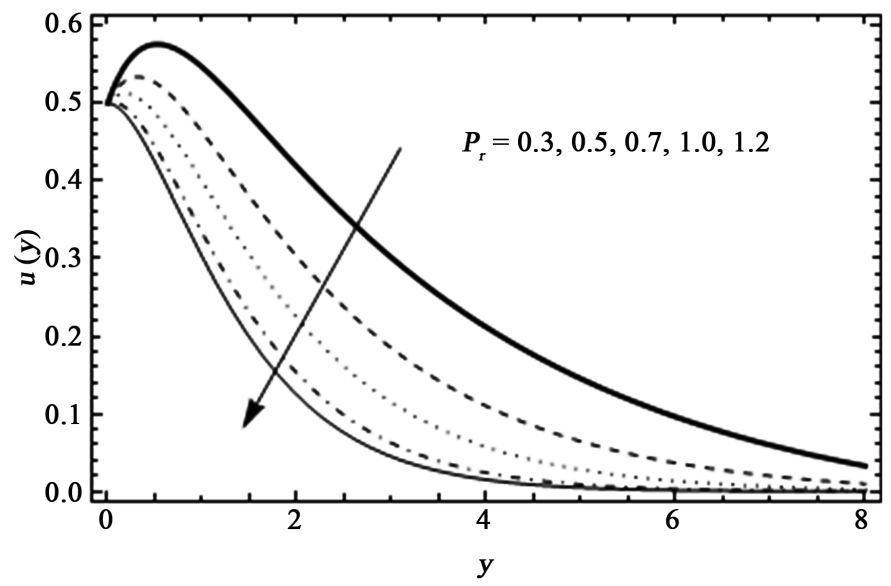

Figure 10. Velocity profile for different values of $P_{r}$ $\lambda=5.0, G_{r}=2.0, G_{m}=1.0, \beta=1.0, M=2.0, \eta=1.0, S_{c}=2.0$, $\gamma_{1}=0.2, n=0.1, U=0.5, n_{1}=0.5, \varepsilon=0.01, t=1.0$. 


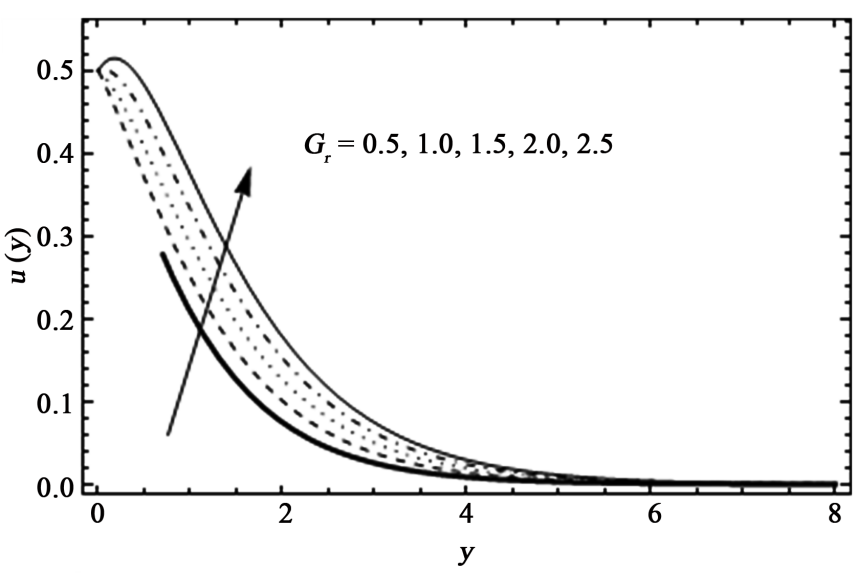

Figure 11. Velocity profile for different values of $G_{r}$ $\lambda=5.0, G_{m}=1.0, \beta=1.0, M=2.0, \eta=1.0, P_{r}=1.0, S_{c}=2.0$, $\gamma_{1}=0.2, n=0.1, U=0.5, n_{1}=0.5, \varepsilon=0.01, t=1.0$.

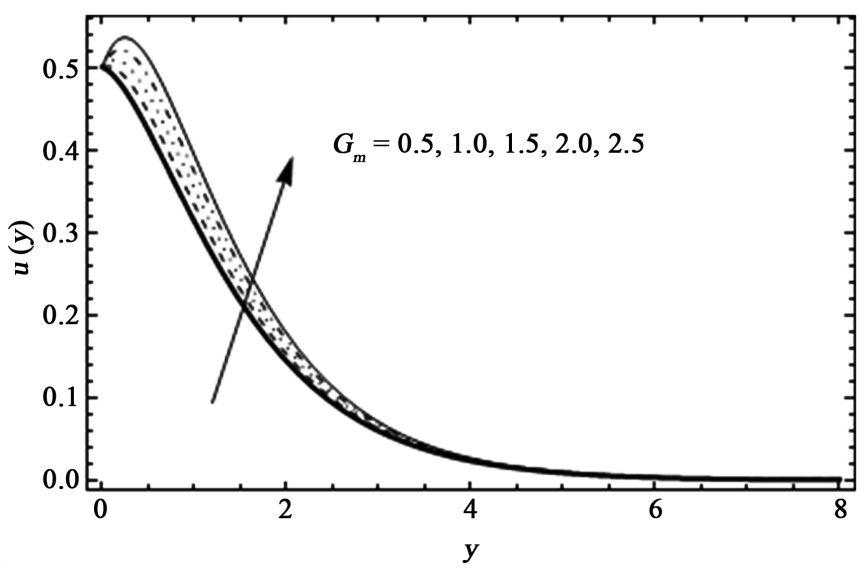

Figure 12. Velocity profile for different values of $G_{m}$ $\lambda=5.0, G_{r}=2.0, \beta=1.0, M=2.0, \eta=1.0, P_{r}=1.0, S_{c}=2.0$, $\gamma_{1}=0.2, n=0.1, U=0.5, n_{1}=0.5, \varepsilon=0.01, t=1.0$.

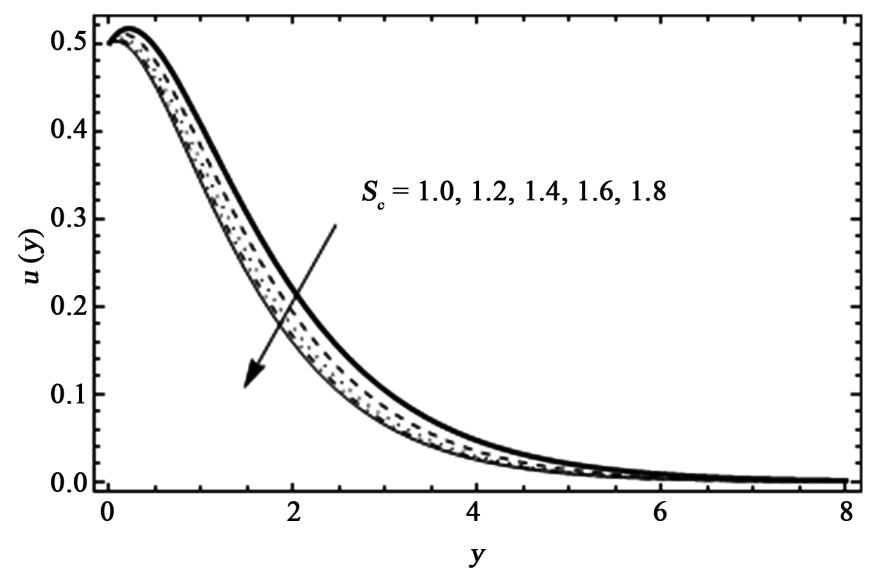

Figure 13. Velocity profile for different values of $S_{c}$ $\lambda=5.0, G_{r}=2.0, G_{m}=1.0, \beta=1.0, M=2.0, \eta=1.0, P_{r}=1.0$, $\gamma_{1}=0.2, n=0.1, U=0.5, n_{1}=0.5, \varepsilon=0.01, t=1.0$. 
profiles is accompanied by simultaneous reductions in the momentum boundary layer thickness.

\section{Conclusions}

The mass and heat transfer of a two-dimensional oscillatory, viscous, electrically conducting fluid over an infinite vertical moving permeable plate in a saturated porous medium with the presence of a transverse magnetic field and chemical reaction was studied. It was found that, increasing the permeability parameter and the chemical reaction parameter will lead to an increase in the fluid velocity. The velocity distribution increases as the magnetic field parameter decreases.

The concentration decreases as the Schmidt number increases, while increases as chemical reaction parameter increases, the fluid velocity and concentration increase with time. Near the surface, the velocity profile increases to the maximum and then decreases until it reaches an asymptotic value.

As time decreases, the thermal boundary layer thickness decreases and the temperature gradient at the wall increases and hence the heat transfer rate increases. The temperature profile is large at the surface and then decreases far away from the wall until it reaches an asymptotic value, the velocity and temperature of the flow field decreases as Prandtl number increases.

Increasing in the thermal Grashof number leads to a rise in the velocity.

Finally the fluid velocity decreases as the Schmidt number increases; the reduction in velocity profiles is accompanied by simultaneous reductions in the momentum boundary layer thickness.

\section{References}

[1] Ibrahim, F.S., Elaiw, A.M. and Bakr, A.A. (2008) Effect of the Chemical Reaction and Radiation Absorption on the Unsteady MHD Free Convection Flow Past a Semi Infinite Vertical Permeable Moving Plate with Heat Source and Suction. Communications in Nonlinear Science and Numerical Simulation, 13, 1056-1066. https://doi.org/10.1016/j.cnsns.2006.09.007

[2] Vajravelu, K. (1986) Hydrodynamic Flow and Heat Transfer over Continuous, Moving Porous, Flat Surface. Acta Mechanica, 64, 179-185. https://doi.org/10.1007/BF01450393

[3] Rout, B.R., Parida, S.K. and Panda, S. (2013) MHD Heat and Mass Transfer of Chemical Reaction Fluid Flow over a Moving Vertical Plate in Presence of Heat Source with Convective Surface Boundary Condition. International Journal of Chemical Engineering, 2013, 10. https://doi.org/10.1155/2013/296834

[4] Modather, M., Rashas, A.M. and Chamkha, A.J. (2009) An Analytical Study of MHD Heat and Mass Transfer Oscillatory Flow of a Micropolar Fluid over a Vertical Permeable Plate in a Porous Medium. Turkish Journal of Engineering and Environmental Sciences, 33, 245-257.

[5] Reddy, S.K., Kesavaiah, D.C. and Raja Shekar, M.N. (2013) MHD Heat and Mass Transfer Flow of a Viscoelastic Fluid Past an Impulsively Started Infinite Vertical Plate with Chemical Reaction. International Journal of Innovative Research in Science, Engineering and Technology, 2, 973-981.

[6] Das, U.N., Deka, R.A. and Soundalgekar, V.M. (1994) Effect of Mass Transfer on 
Flow Past an Impulsively Started Infinite Vertical Plate with Constant Heat Flux and Chemical Reaction. Forschung im Ingenieurwesen, 60, 284-287. https://doi.org/10.1007/BF02601318

[7] Ahmed, N. and Das K. (2013) MHD Mass Transfer Flow Past a Vertical Porous Plate Embedded in a Porous Medium in a Slip Flow Regime with Thermal Radiation and Chemical Reaction. Open Journal of Fluid Dynamics, 3, 230-239. https://doi.org/10.4236/ojfd.2013.33028

[8] Muthucumaraswamy, R. and Ganesan. P. (2001) First Order Chemical Reaction on Flow Past an Impulsively Started Vertical Plate with Uniform Heat and Mass Flux. Acta Mechanica, 147, 45-57. https://doi.org/10.1007/BF01182351

[9] Muthucumaraswamy, R. (2002) Effect of a Chemical Reaction on a Moving Isothermal Vertical Surface with Suction. Acta Mechanica, 155, 65-70.

https://doi.org/10.1007/BF01170840

[10] Kandasamy, R., Md. Raj, A.W.B. and Khamis, A.B. (2006) Effects of Chemical Reaction, Heat and Mass Transfer on Boundary Layer Flow over a Porous Wedge with Heat Radiation in the Presence of Suction or Injection. Theoretical Applied Mechanics, 33, 123-148. https://doi.org/10.2298/TAM0602123K

[11] Anjali Devi, S.P. and Kandasamy, R. (2002) Effects of Chemical Reaction, Heat and Mass Transfer on Non-Linear MHD Laminar Boundary Layer Flow over a Wedge with Suction or Injection. International Communications in Heat and Mass Transfer, 29, 707-716. https://doi.org/10.1016/S0735-1933(02)00389-5

[12] Chamkha, A. (2003) MHD Flow of Uniformly Stretched Vertical Permeable Surface in the Presence of Heat Generation/Absorption and a Chemical Reaction. International Communications in Heat and Mass Transfer, 30, 413-422. https://doi.org/10.1016/S0735-1933(03)00059-9

[13] Kandasamy, R., Periasamy, K. and Sivagnana Prabhu, K.K. (2005) Chemical Reaction, Heat and Mass Transfer on MHD Flow over a Vertical Stretching Surface with Heat Source and Thermal Stratification Effects. International Journal of Heat and Mass Transfer, 48, 4557-4561. https://doi.org/10.1016/j.ijheatmasstransfer.2005.05.006

[14] Khadrawi, A.F. and Al-Odat, M.O. (2005) Transient MHD Free Convection Flow over a Permeable Vertical Moving Plate Embedded in Porous Medium with Uniform Suction. International Journal of Heat and Technology, 23, 81-87.

[15] Chamkha, A.J. (1997) MHD Free Convection from a Vertical Plate in Saturated Porous Medium. Applied Mathematical Modelling, 21, 603-609. https://doi.org/10.1016/S0307-904X(97)00084-X

[16] Aldoss, T.K., Al-Nimr, M.A., Jarrah, M.A. and Al-Sha'er, B.J. (1995) Magnetohydrodynmic Mixed Convection from Vertical Plate Embedded in Porous Medium. Numerical Heat Transfer, Part A, 28, 635-645. https://doi.org/10.1080/10407789508913766

[17] Bejan, A. and Kraus, A.D. (2003) Heat and Mass Transfer Handbook. John Wiley and Sons, Hoboken.

[18] Incropera, P., De Witt, D.P., Bergman, T.L. and Lavine, A.S. (2007) Introduction to Heat Transfer. 5th Edition, Wiley, Hoboken.

[19] Holman, J.P. (2009) Heat Transfer. 10th Edition, McGraw-Hill, New York. 